\title{
Relevo E Edificações Tridimensionais Usando Fractais
}

\author{
Professor Doutor Jason Paulo Tavares Faria Junior (Sistemas da Informação e Engenharia \\ Ambiental - UniFOA) - jason-rj@bol.com.br
}

\section{Resumo}

Neste trabalho será apresentado o desenvolvimento de um software que permite a visualização tridimensional de mapas cartográficos bidimensionais utilizando fractais para a representação geométrica de relevos e edificações. Envolvendo várias disciplinas como computação gráfica, matemática, Topografia, linguagem de programação e física. Este projeto é orientado aos alunos dos últimos anos dos cursos de graduação e de iniciação científica com o objetivo de desenvolver este procedimento automatizado com a finalidade de comercialização.

Palavras chave: Relevo; Edificações; Fractais

\section{Introdução}

A Geometria dos Fractais, trouxe contribuições para a análise de estruturas complexas existentes na natureza, não explicáveis pela tradicional Geometria. Assim, foi criado um número para representar dimensões intermediárias entre as bem conhecidas da Geometria : ele é a dimensão fractal, que mede o grau de aspereza, fragmentação ou de irregularidade de um objeto. A medição da precipitação pode ser feita em diversos segmentos temporais, os quais compõem estruturas e representam objetos que podem ser analisados pelo uso da abordagem fractal. Fractais são operações geométricas aplicadas repetidamente visando um resultado gráfico, como a obtenção do efeito de um relevo tridimensional (figura 1).

A partir da década de 70 do século passado, uma nova abordagem de análise tem tido espaço no campo da Matemática e assim como nas ciências naturais, sociais e aplicadas; tratase da Geometria dos Fractais, que está em sintonia com a Teoria do Caos. Esta geometria teve seu desenvolvimento a partir da década de 60, pelo polonês Benoit Mandelbrot e foi publicada tardiamente 1975 na obra Les Objects fractals: forme, Hasard et Fimension e, traduzido para o inglês em 1977 com o titulo, Fractals: Form, chance, and Dimension que, posteriormente, foi reeditado com o nome de The Fractal Geometry of Nature, a qual lhe deu fama internacionalmente.

Mandelbrot aplica o termo fractal (originário da palavra fractus, que é derivada do verbo frangere que significa quebrar, fraturar em latim) para adjetivar a teoria que buscava explicar melhor as origens da ordem e da desordem da natureza. Segundo Guerrini (1996) "fractais são os contornos do caos. Fractais são objetos que apresentam auto-semelhança e complexidade infinita, ou seja, sempre pela interação de processos simples”. Por conseguinte, a Geometria Fractal também deve ser aplicada à Geografia, pois, esta se preocupa com a disposição e o arranjo das estruturas espaciais.

A abordagem do tema justifica-se, pela necessidade de obter uma visão tridimensional, dos relevos e das edificações através mapas que na maioria das vezes são bidimensionais. Adquirir esta tecnologia e a elaboração de um software nesta área, permitirá o 
desenvolvimento de projetos e simulações mais precisas por parte dos engenheiros, geólogos, arquitetos e na área da educação, onde alunos dos cursos de engenharia civil, geografia entre outros, poderão ter um melhor entendimento das curvas de nível dos mapas cartográficos 2D, já que atualmente usa-se para melhor entendimento destes, maquetes em escalas apropriadas, porém este é um processo demorado e mais caro do que o procedimento automatizado proposto neste trabalho.

\section{Teoria do Caos e Geometria dos Fractais}

A Geometria dos Fractais surge como controvérsia quando comparado com a tradicional Geometria Euclidiana. Essa abordagem vem ganhando importância na Matemática e nas ciências naturais, sociais e tecnológicas tendendo a explicar de forma mais precisa as origens da ordem e da desordem, baseando-se nos comportamentos caóticos dos sistemas, onde o determinismo restrito e o desenvolvimento aparentemente aleatório, de forma acidental andam juntos, formando a regra da natureza. Portanto, "a nova geometria espelha um universo que é irregular, e não redondo; áspero, e não liso. È uma geometria das reentrâncias, de pressões, do que é fragmentado, torcido, emaranhado e entrelaçado" (Gleik, 1990) Com isto, o entendimento da complexidade da natureza espera que a complexidade não é apenas algo aleatório.

A Teoria do Caos, elaborada através das pesquisas realizadas por Edward Lorenz em 1961 demonstra que "dado um conhecimento aproximado das condições iniciais de um sistema e um entendimento da lei natural, pode-se calcular o comportamento aproximado desse sistema” (Gleik, 1990). Assim, Lorenz desenvolveu interesse pela meteorologia e vontade de mostrar um simulador capaz de fazer previsões do tempo, fazendo observações importantes para a formulação da Teoria do Caos.

Para este mesmo autor as repetições da natureza têm um padrão, com alterações, formando uma "desordem ordenada". Existia uma ligação entre a periodicidade e a imprevisibilidade e que influências muito pequenas, quando postas de lado, tinham um grande efeito no resultado final. Esta conclusão é conhecida como "Efeito Borboleta", ou seja, uma cadeia de acontecimentos pode ter um ponto de crise que aumenta pequenas mudanças. Mas, o Caos significa que tais pontos estão por todas as partes. As equações lineares não eram suficientes para explicar as recentes indagações de Lorenz. Por isso, ele procurou expressões matemáticas demonstrando relações que não eram rigorosamente proporcionais. Tornou-se famoso por isto, mas, só depois de alguns anos que uma geometria foi desenvolvida e acabou se aliando com os princípios da Teoria do Caos.

“Ao criar a geometria fractal, Benoit Mandelbrot (1982) estabelecia as bases para o estudo focalizando as formas fragmentadas, fraturadas, rugosas e irregulares. Tais categorias de formas são normalmente geradas por uma dinâmica caótica, de modo que a geometria fractal descreve os traços e as marcas deixadas pela passagem dessa atividade dinâmica." Christofolleti (1997).

Os fractais para Briggs (1992) e Christofolleti (1997) "descrevem a rugosidade do Mundo, sua energia, suas mudanças e transformações dinâmicas. Os fractais são imagens das maneiras pelas quais as coisas se entrelaçam e se separam, retroalimentando-se em cada outra em si mesmas.” Portanto, os fractais estão em nosso meio: árvores, montanhas, nós mesmos, enfim, todas estas demonstrações são sinais da atividade dinâmica trabalhando.

Para que seja possível o estudo dos objetos e atribuindo-lhes dimensionalidades mais precisas, a Geometria dos Fractais é a mais correta, pois, a Geometria dimensional clássica apenas descreve os objetos considerando a uni (linhas), bi (áreas) e tridimensionalidade 
(volumes) em números inteiros. Todavia, "a descrição dimensional em números inteiros dos objetos espaciais é questão de conveniências, não um atributo fundamental do Universo. Em conseqüência, a dimensão fracionada de um objeto que descreve a sua rugosidade é uma ampliação útil de um conjunto de definições sobre a estrutura dimensional. Portanto, a dimensão fractal é uma descrição útil das estruturas espaciais” Christofolleti (1997).

Os fractais possuem três princípios: escalante ("scalling”), auto-similaridade, aleatoriedade. A primeira significa que os fractais em escalas diferentes apresentam os mesmos detalhes. Este princípio vem acompanhado da auto-similaridade: nas diversas escalas ela tem a mesma forma ou semelhança, ou seja, simetria entre as escalas. A aleatoriedade afirma que o Caos não é uma ausência completa de padrões, mas, está sujeito a algumas regras, mesmo que o resultado final seja previsível.

É importante ressaltar que dois objetos são similares se eles tem a mesma forma, independentemente se os tamanhos são diferentes, mas, deve-se ter a mesma proporcionalidade entre os segmentos e os ângulos devem ser iguais. O fator de ampliação de objetos é chamado de fator de escalante ("scalling fator") e a transformação entre os objetos é chamada transformação de similaridade.

\section{O Protótipo}

Inicialmente foi desenvolvido um protótipo (figura 1) que gera relevos 3D através de alturas aleatórias de três vértices de um triângulo equilátero (figura 2), então encontra-se o ponto médio de cada lado do triângulo criando assim um novo triângulo interno. Aplicando este mesmo procedimento aos novos triângulos, cria-se uma representação gráfica de um relevo real (figura 3).

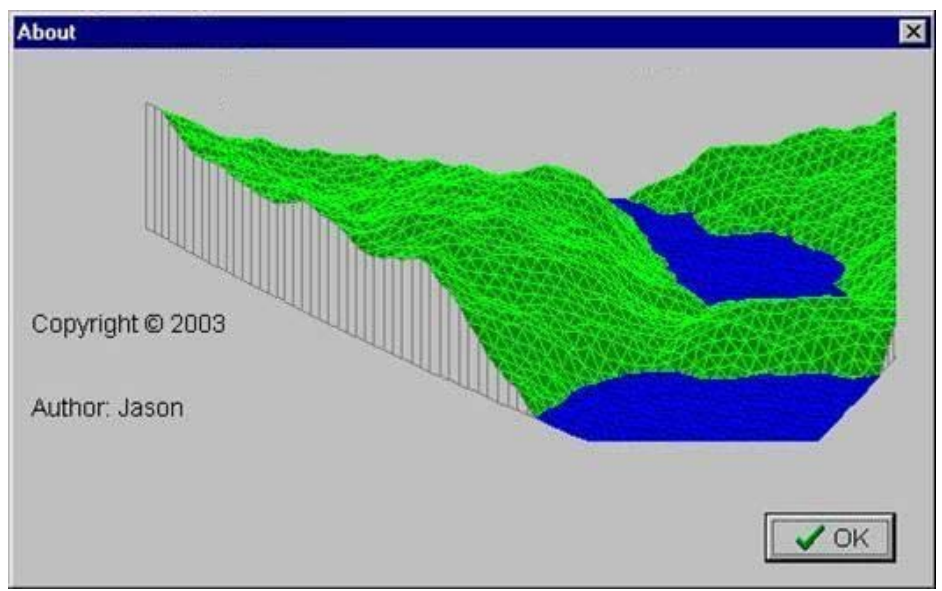

Figura 1- Tela "About" do Software 


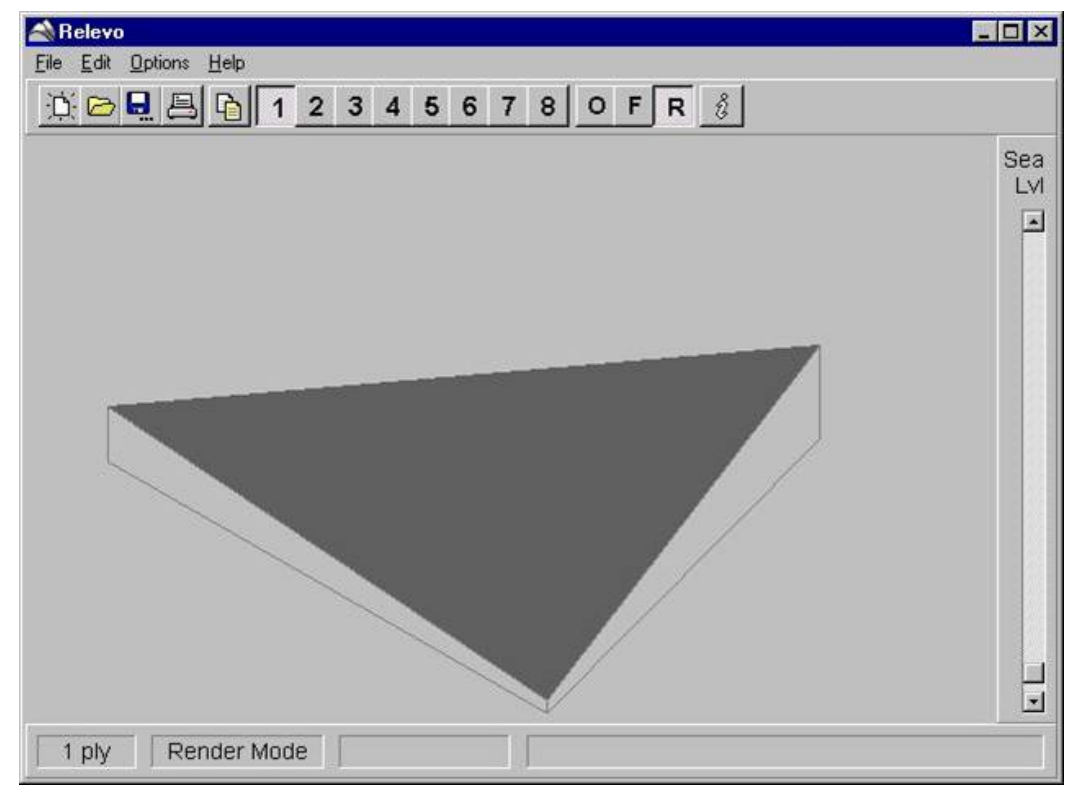

Figura 2-Três vértices de um triângulo equilátero.

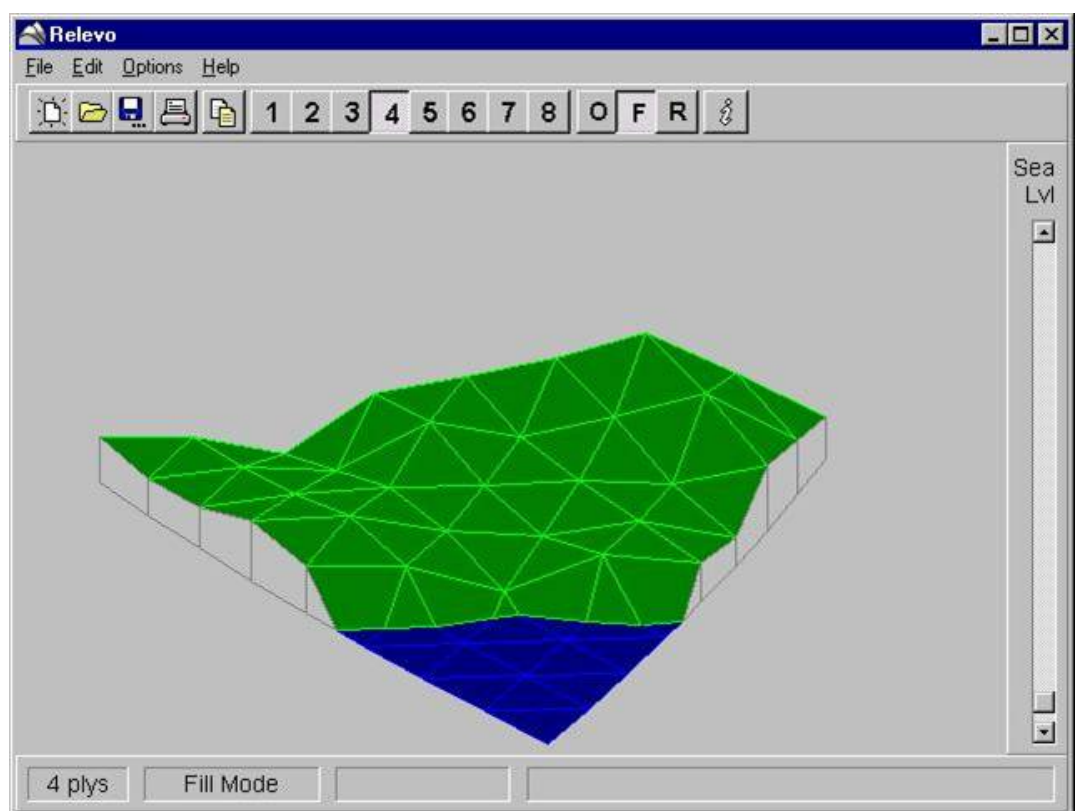

Figura 3- Aplicando um mesmo procedimento aos novos triângulos, cria-se uma representação gráfica de um relevo real

\section{Implementação do Programa de Computador}

O programa que está sendo desenvolvido nesse trabalho, implementa a técnica de Fractais e cenários tridimensionais.

A interface gráfica para o usuário foi projetada para operar com o sistema operacional "Windows" e utiliza tela de apresentação , caixas de diálogo, interfaces de visualização dos cenários. Mapas poderam ser visualizados após serem importados por arquivos gráficos disponíveis comercialmente Google Earth) ou por digitalização (Scaner).

Os mapas digitalmente obtidos poderão ser carregados no programa através de um arquivo de extensão .BMP, .WMF ou .ICO. Esses formatos permitem que ambientes reais 
gerados em digitalização de mapas e "softwares" comerciais, sejam transportados para o programa .

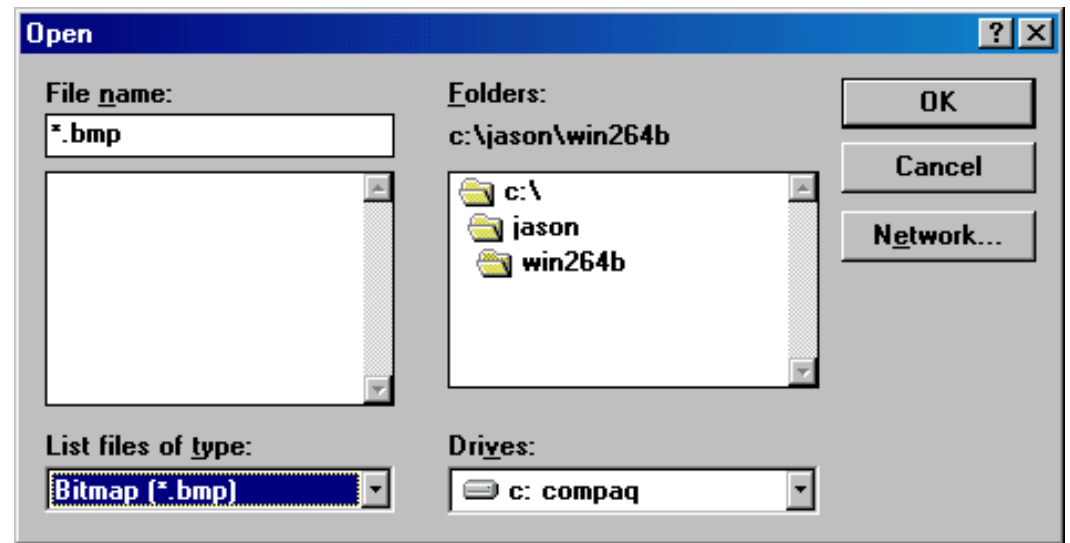

Figura 4- Caixa de Diálogo para a inserção dos mapas

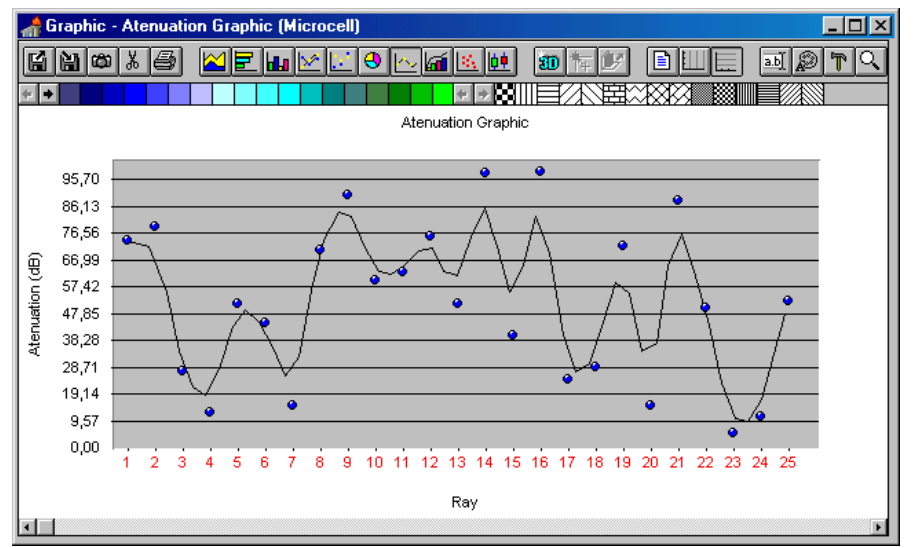

Figura 5- Gráfico do Relevo Bidimensional

Estes gráficos possuem uma barra de ferramentas que permite ao usuário:

- Copiar, recortar e imprimir o gráfico

- Visualizar o gráfico em três ou duas dimensões

- Escolher outros tipos de gráficos ( barra, área, linha, pontos, etc.) 


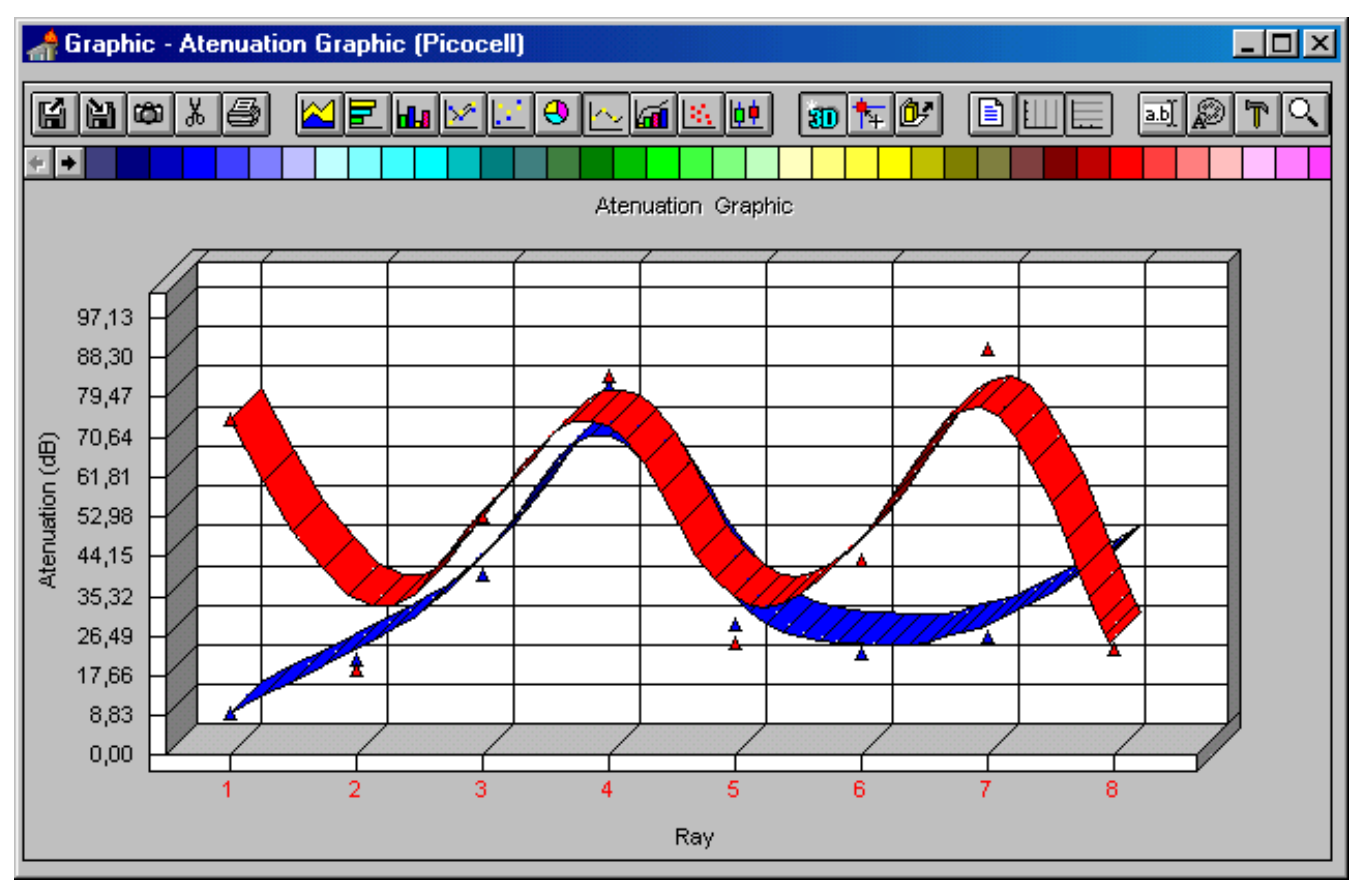

Figura 6- Gráfico de 2 Relevos

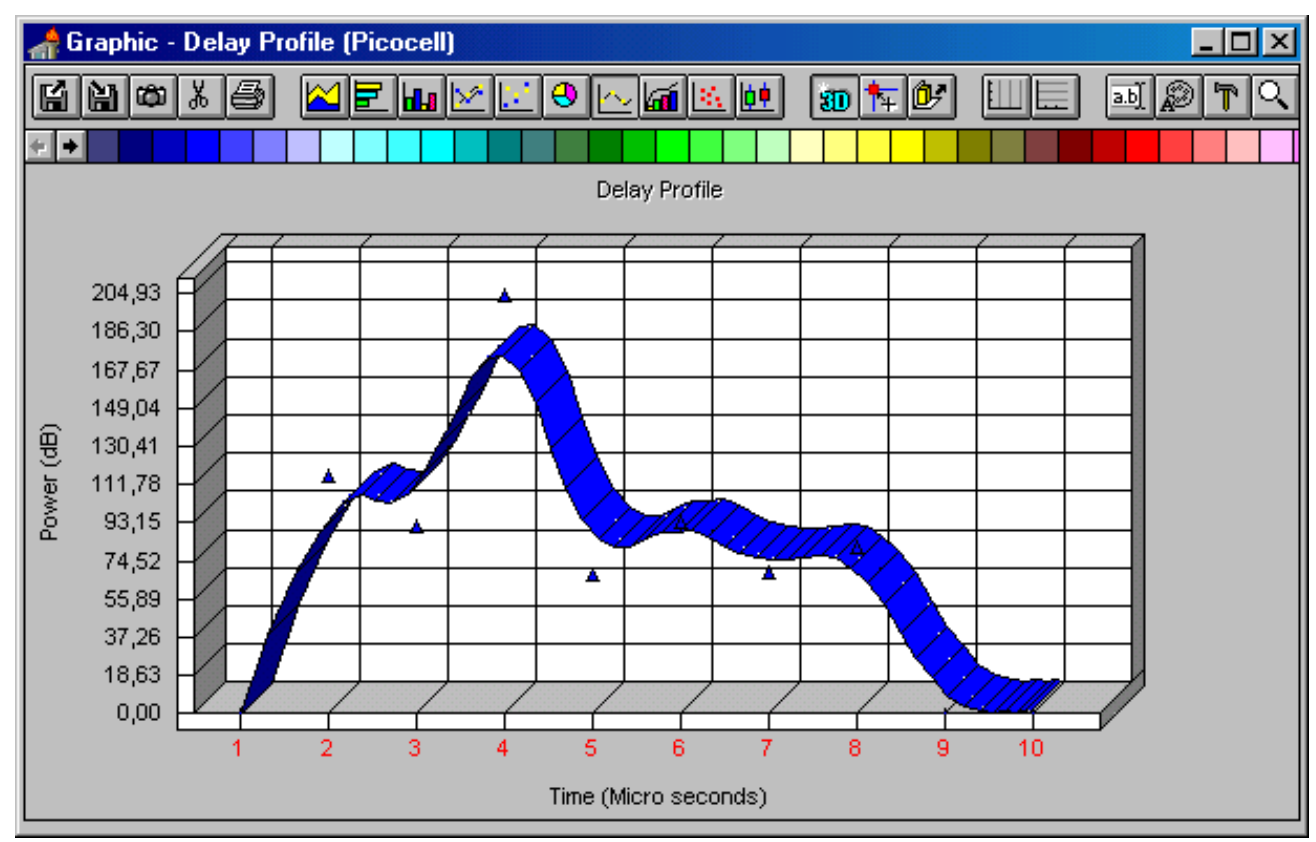

Figura 7- Gráfico de um Relevo

Áreas Urbanas também serão visualizadas em versões futuras do Software.As alturas das edificações são descriminadas por cores formando assim um cenário tridimensional apesar da visualização de topo dos mapas. A tela de simulação também possui uma barra de menus, onde o usuário tem acesso aos formulários de entrada de dados, visualização dos gráficos e etc.. 


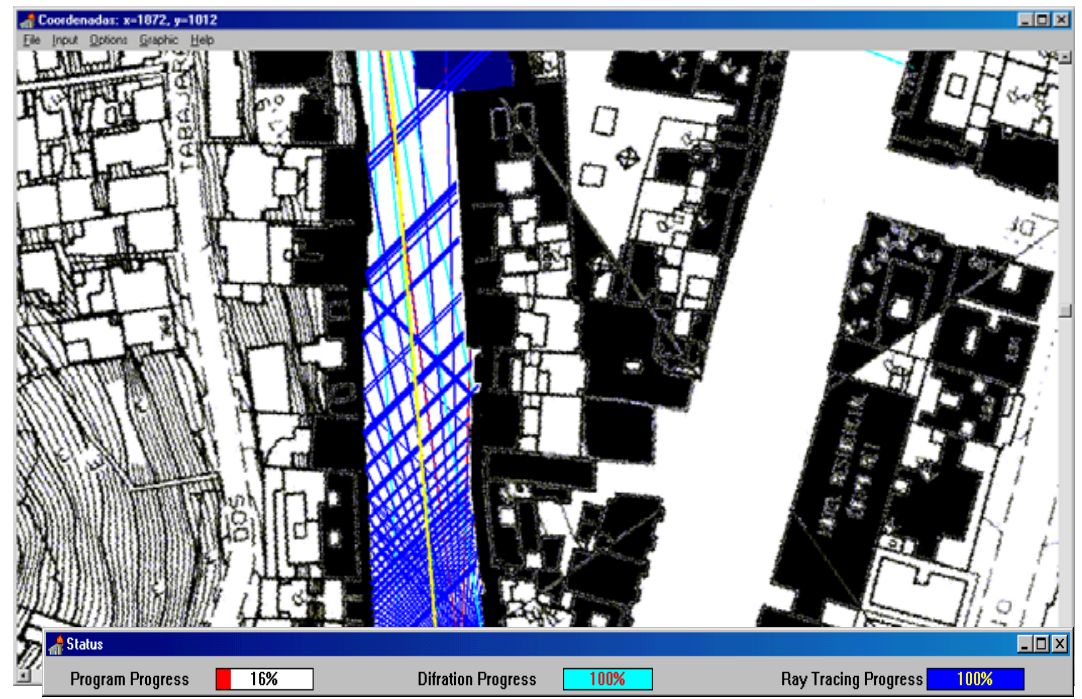

Figura 8- Interface de visualização do cenário urbano

A interface de visualização do cenário permite que o cenário seja expandido e conseqüentemente fica possível verificar um maior número de construções, bastando o usuário selecionar o menu “Options” e depois “Expand”. Ainda há os objetos denominados "scroll bars” que permitem ao usuário navegar pelos mapas.

Um outro recurso disponível são os objetos "gauge” que fornecem informações sobre o progresso do programa, assim durante o processo de visualização, que pode durar alguns segundos, o usuário pode verificar quanto tempo falta para terminar o processo.

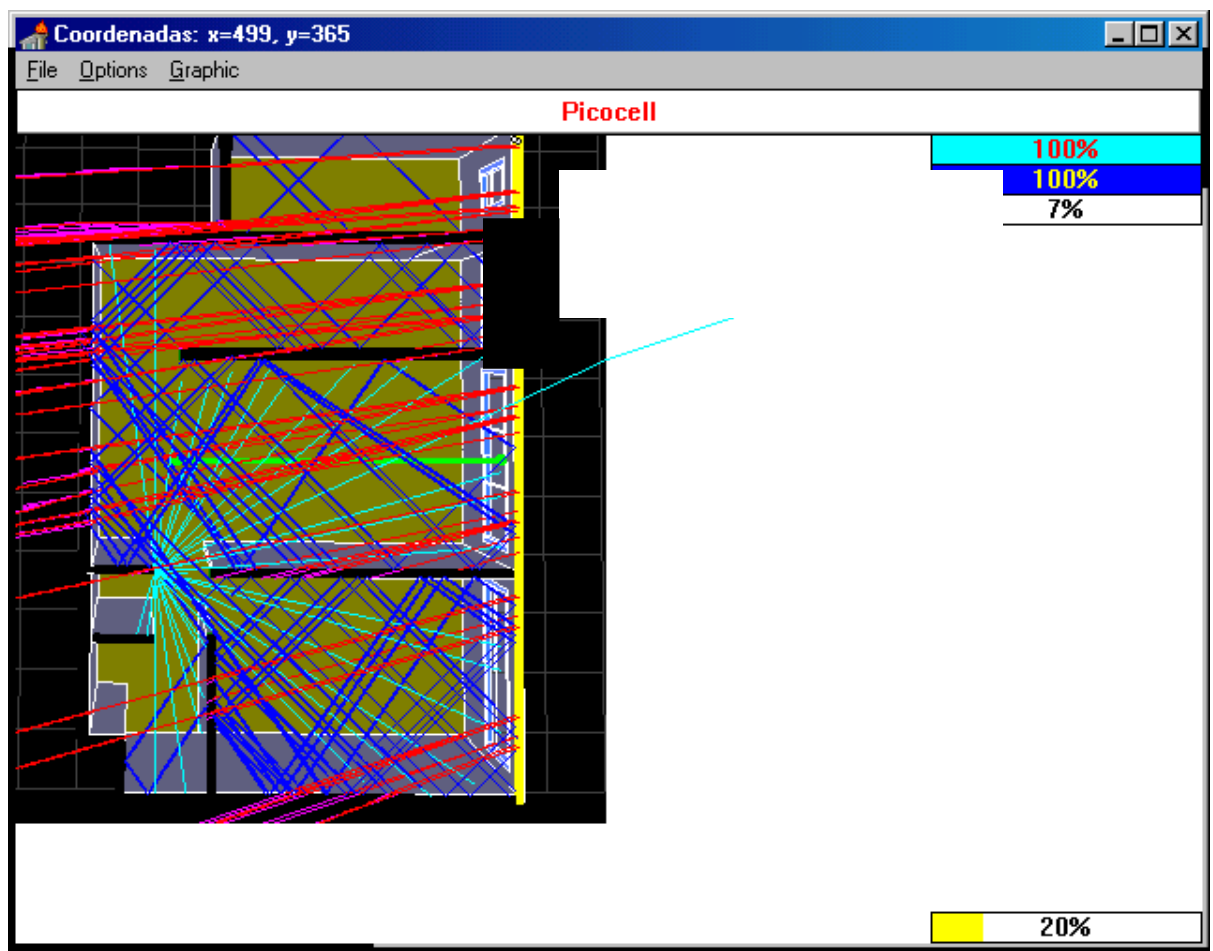

Figura 9- Ambiente “indoor”

\section{Descrição do Cenário}


Não há uma forma definitiva que descreva como é gerado um cenário. A princípio, o cenário deve ser o mais próximo possível do ambiente por ele representado. Porém, ambientes reais são complexos, com obstáculos das mais variadas formas e construídos com diversos tipos de materiais. Deve-se buscar, ao construir o cenário, atender a um compromisso entre fidelidade na descrição do ambiente e praticidade de implementação do cenário.

A forma usual de se criar um cenário em três dimensões (3D) é através da representação dos componentes do ambiente através de poliedros, como ilustra a Figura 3.

A descrição geométrica consiste em compor o cenário através de sólidos multifacetados e de seções de planos. Dessa forma, todos os obstáculos relevantes do ambiente são transformados em polígonos (as faces dos poliedros).

O dimensionamento, a rotação e a translação de um objeto tridimensional formado por polígonos envolve a transformação das coordenadas dos vértices. Cada operação é um tipo de transformação de coordenadas tridimensionais

\section{Considerações Finais}

Este software esta sendo desenvolvido nas linguagens Delphi e Assembly. O uso de fractais tem como objetivo criar uma representação gráfica de um relevo real. Envolvendo várias disciplinas como computação gráfica, matemática, cartografia, linguagem de programação e física. Este projeto é orientado aos alunos dos últimos anos dos cursos de graduação e de iniciação científica com o objetivo de publicar artigos em congressos e desenvolver este procedimento automatizado com a finalidade de comercialização.

\section{Referências}

CHRISTOFOLETTI, A. L. H. Análise fractal e multifractal da estrutura de estações chuvosas em localidades do estado de São Paulo. Rio Claro: IGCE/ Unesp. (Tese de Doutorado). 1997 .

GLEICK, J. - Caos: A Criação de uma Nova Ciência. Rio de Janeiro: Editora Campus, 1990.

FEDER, J. Fractals. Nova Yorque. Plenum Press. 1988.

GUERRINI, I. A. - Caos e Fractais em Física Aplicada. Botucatu: IB/ Unesp, 1996

HURST, H. E. - Lont-term storage capacity of reservois. Procced. American Soc. Civil Engineering. N 116. páginas 770-808. 1951.

LORENZ, E .N. - A esencia do caos. Brasilia. Editora Universidade de Brasilia. 1996. 
MONTEIRO, C. A. de F. - A Frente Polar Atlântica e as chuvas de inverno na fachada SulOriental do Brasil. São Paulo: IG/USP (Séries Teses e Monografías, n. 1)., 1969

MONTEIRO, C. A. de F. - A dinâmica climática e as chuvas no Estado de São Paulo: estudo geográfico sob a forma de Atlas. São Paulo: IG/USP, 1973.

PEREIRA JUNIOR, A. - Análise fractal da distribuição espacial das chuvas no Estado de São Paulo. Rio Claro: IGCE/Unesp, 1998.

\section{Informações bibliográficas:}

Conforme a NBR 6023:2002 da Associação Brasileira de Normas Técnicas (ABNT), este texto científico publicado em periódico eletrônico deve ser citado da seguinte forma:

FARIA JUNIOR, J. P. T.. Relevo E Edificações Tridimensionais Usando Fractais. Cadernos UniFOA, Volta Redonda, ano 1, $\mathrm{n}^{\circ} .1$, jul. 2006. Disponível em:

<http://www.unifoa.edu.br/pesquisa/caderno/edicao/01/33.pdf > 